\title{
THE EFFECT OF $\gamma$-AMINOBUTYRIC ACID ON BLOOD PRESSURE
}

\author{
HIDEHIKO TAKAHASHI, MASAKO TIBA, MITSUGU IINO \\ AND TAKESHI TAKAYASU* \\ Department of Physiology, Tokyo Medical College, Tokyo
}

Free $\gamma$-aminobutyric acid which was known to be widely distributed in the plant kingdom was found by Awapara $(1,2)$ and Roberts and Frankel (9) to be present in the brain of various animals. It was also found in the gastric juice (5), in the saliva (8), and in the dog's sciatic nerve (7).

Recent studies $(1,2,9)$ on $\gamma$-aminobutyric acid, especially on its distribution and metabolism, seem to indicate that free $\gamma$-aminobutyric acid exists abundantly. in nervous tissues and that this acid is an intermediate of the metabolism of glutamic acid.

Our attention was called to the fact that $\gamma$-aminobutyric acid is the only $\omega$ amino acid existing free in the animal body, except for $\beta$-alanine, and that this acid is present abundantly in the nerve cell and thus we are performing various experiments with this substance. In the present paper its acute action on blood pressure will be reported.

\section{METHODS}

Rabbits (2-3 kg. of body weight) were used. They were anesthetized with $25 \%$ urethane (1-1.5 g. per kg.) either intramuscularly or intraperitoneally. Blood pressure was recorded with a mercury manometer in the carotid artery. Respiration was recorded with a tambour connected to a tracheal cannula

The compounds to be studied were dissolved in Ringer solution, and 1-2 ml. of the solution were injected intravenously. All the substances used were synthetized preparations.

\section{RESULTS}

The typical action of $\gamma$-aminobutyric acid on blood pressure and respiration

An intravenous injection of $\gamma$-aminobutyric acid (m.p. $196^{\circ} \mathrm{C}$.) caused a fall of blood pressure generally within $10-15 \mathrm{sec}$. in all rabbits. Blood pressure fell abruptly, and gradually returned to the original level. At the same time, bradycardia was observed. The fall of blood pressure lasted from a few minutes to some ten minutes or longer. This duration of the fall was proportional to the concentration of the acid. Respiration was transitorily reduced in frequency at

Received for publication July 26, 1955.

* 高橋日出彥 千葉正子 飯野 貢 高安健之 
first and then increased in frequency and amplitude for a few minutes. A typical experiment with $9.3 \mathrm{mg} / \mathrm{kg}$. $\gamma$-aminobutyric acid is shown in fig. 1 .

The minimal effective dose of $\gamma$-aminobutyric acid on blood pressure of rabbits under urethane anesthesia was $70 \mathrm{r} / \mathrm{kg}$. of body weight. Fig. 2 shows an example of this minimal effect. Without anesthesia, this minimal effective dose was reduced to $50 \mathrm{r} / \mathrm{kg}$. of body weight.

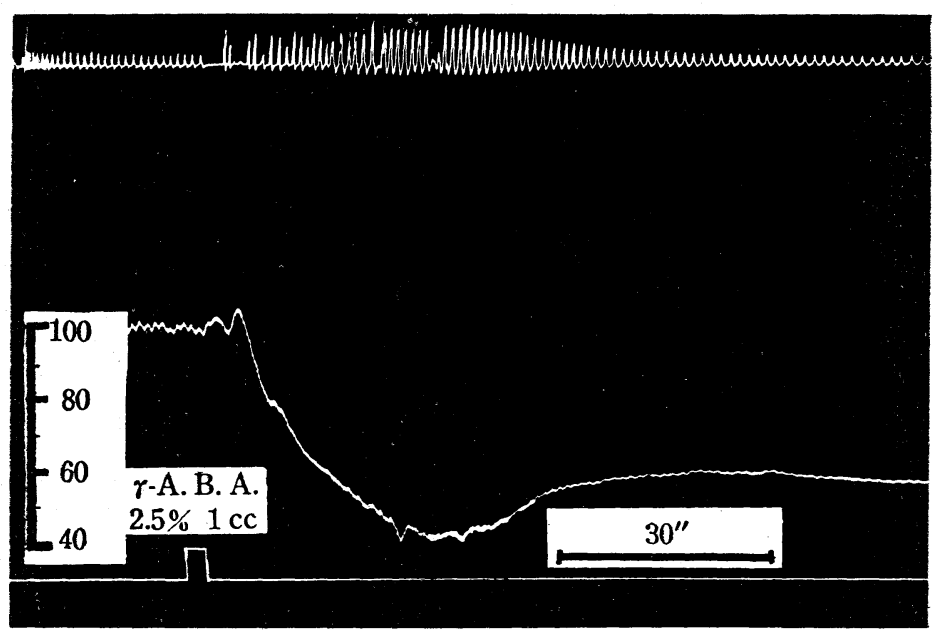

FIG. 1. The effect of $\gamma$-aminobutyric acid on blood pressure and respiration. Rabbit (2.7 kg.) under urethane anesthesia: Room temperature $15^{\circ} \mathrm{C}$. 1 cc. of $2.5 \% \cdot \gamma$-aminobutyric acid was injected in the vein of the ear. The upper curve: respiration.

The lower curve: blood pressure.

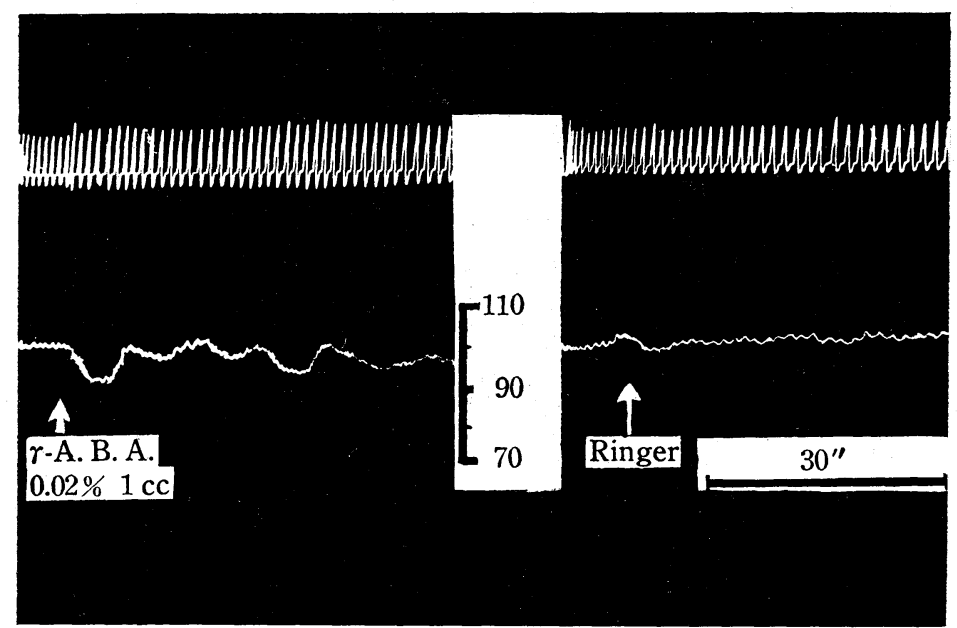

FIG. 2. The effect of $\gamma$-aminobutyric acid of the thresholdal dose on blood pressure. Rabbit $\left(2.9 \mathrm{~kg}\right.$.) under urethane anesthesia : Room temperature $15^{\circ} \mathrm{C}$.

1 cc. of $0.02 \% \gamma$-aminobutyric acid and $1 \mathrm{cc}$. of Ringer solution as control were injected in the vein of the ear. 
Fig. 3 shows the relation between the magnitude of the fall of blood pressure and the concentration of $r$-aminobutyric acid (\% concentration). Each point in this graph shows the average of several experiments.

This graph shows that, when other conditions were almost the same, the magnitude of the fall of blood pressure increased linearly with the logarithm of the concentration of $r$-aminobutyric acid within some limits. But, in higher concentrations, this relation was broken and the effect became rather smaller. Consequently, there was an optimal dose of $r$-aminobutyric acid that caused the most striking effect on blood pressure. In our experiment this optimal dose of this acid was 8-12 mg. per kg. of body weight.

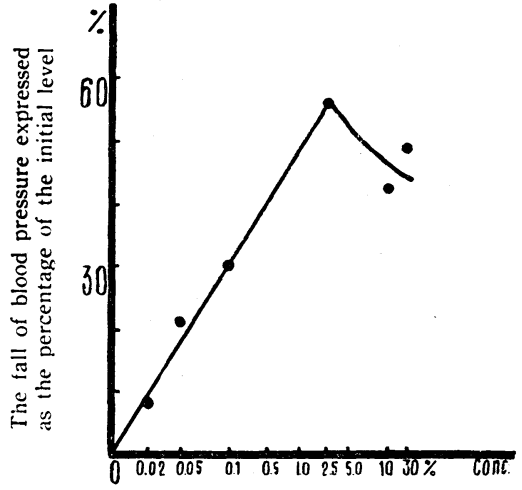

FIG. 3. The relation between the magnitude of the fall of blood pressure and the concentration of $\gamma$-aminobutyric acid. $1 \mathrm{cc}$. of $\gamma$-aminobutyric acid of various concentrations was injected intravenously in rabbits under urethane anesthesia.

The effect of $\gamma$-aminobutyric acid on blood pressure was variable in magnitude according to conditions of rabbits. Among these conditions, the initial level of blood pressure was a predominant factor. Thus, in the case where the initial level of blood pressure was high, the fall of blood pressure by $r$-aminobutyric acid was larger, and vice versa. Even in the case of a very low initial level of blood pressure, a fall of blood pressure by $\gamma$-aminobutyric acid still occurred, although the magnitude of the fall was small, and there never occurred a reversal phenomenon. The values of falls expressed as percentages of initial magnitudes (the magnitude of the maximal fall/the initial magnitude of pressure $\times 100)$ were rather constant, whatever the initial levels were.

\section{The effect of $r$-aminobutyric acid and urethane anesthesia}

In the case of lower concentrations, the effect of $r$-aminobutyric acid on blood pressure was almost the same, whether the rabbit was under urethane anesthesia or not. But, in the case of very high concentrations (10-30\%), remarkable differences were observed between both cases.

In rabbits under urethane anesthesia, $r$-aminobutyric acid of high concentrations (10-30\%) caused a striking fall of blood pressure, lasting not rarely for 1-2 hrs.

In rabbits without anesthesia, this acid of high concentrations caused a transient fall of blood pressure lasting for some ten seconds or 1-2 minutes. Then a rise of pressure followed this fall. This rise of pressure was sometimes a little smaller in magnitude than the 1st transient fall, but the duration was much longer (for a few minutes to 20 minutes).

Such a rise of pressure following the fall was sometimes observed with an injection of $2 \mathrm{cc}$. of $10 \% \gamma$-aminobutyric acid. With 2 cc. of $30 \% \gamma$-aminobutyric acid this phenomenon occurred almost without exception. 
Fig. 4 shows the difference of the effect of $r$-aminobutyric acid on blood pressure between a rabbit under urethane anesthesia and a rabbit without anesthesia.

FIG. 4. The effect of urethane anesthesia on the action of $\gamma$-aminobutyric acid of high concentration on blood pressure.

A. Rabbit (2.3 kg.) under urethane anesthesia. R.T. $22^{\circ} \mathrm{C}$.

$3 \mathrm{cc}$. of $30 \% \gamma$-aminobutyric acid were injected intravenously.

B. Rabbit ( $2.5 \mathrm{~kg}$.) without anesthesia. R.T. $22^{\circ} \mathrm{C}$.

2 cc. of $30 \% \gamma$-aminobutyric acid were injected intravenously. (Time mark 6 sec.)
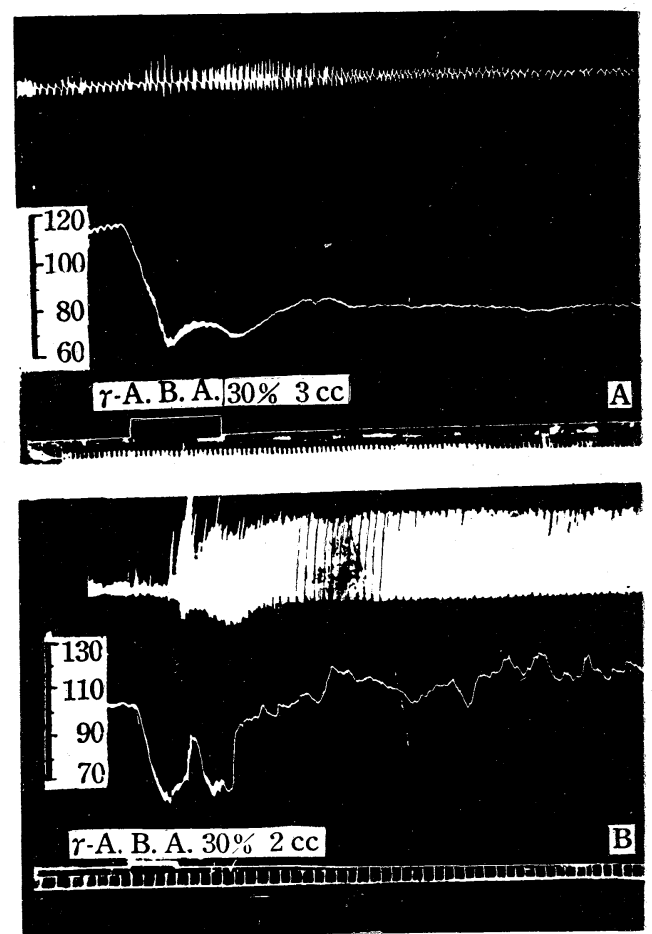

\section{Effects of other $\omega$-amino acids on blood pressure}

Among other $\omega$-amino acids, $\beta$-alanine (decomp. $195^{\circ} \mathrm{C}$.) acts on blood pressure similarly as $\gamma$-aminobutyric acid. But, the thresholdal dosis of $\beta$-alanine $(5 \mathrm{mg} / \mathrm{kg}$.) was 70 times larger than that of $\gamma$-aminobutyric acid. Its power of lowering blood pressure was 100 times smaller that of $\gamma$-aminobutyric acid.

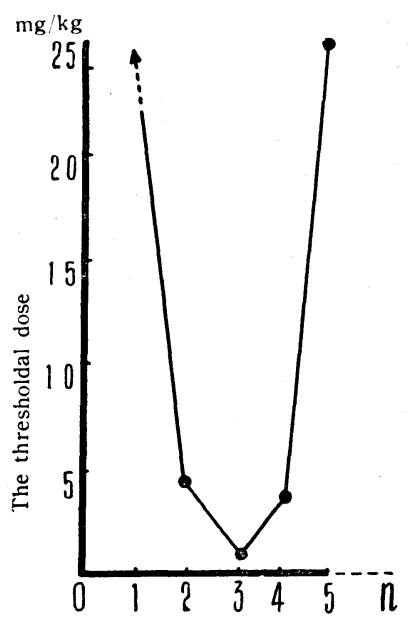

FIG. 5. The relation between the distance of carboxylic group and amino-group of amino acids and their thresholdal doses. In this and the next figure numbers of carbon atoms between carboxylic group and amino-group in the abscissa. 1-glycine, 2 - $\beta$-alanine, 3- $\gamma$-aminobutyric acid, $4-\delta$-aminovaleric acid, 5- $\varepsilon$-aminocaproic acid. 
$\delta$-amino- $\boldsymbol{n}$-valeric acid, [its $\mathrm{HCl}$ salt (m.p. $71-72.5^{\circ} \mathrm{C}$. Fluka Co.) was used] too, caused a fall of blood pressure, with a thresholdal dosis $(3 \mathrm{mg} / \mathrm{kg}$.) 40-fold larger than that of $\gamma$-aminobutyric acid.

$\varepsilon$-aminocaproic acid (m.p. $203^{\circ} \mathrm{C}$.) had only a slight effect on blood pressure, and glycine (decomp. $233^{\circ} \mathrm{C}$.) had no effect.

Fig. 5 shows the relation between the distance of carboxylic group and amino-group of amino acids and their thresholdal doses.

Fig. 6 shows the relation between the distance of two groups of amino acids and their lowering powers at the concentration of $1 / 4$ mole. At this concentration, little difference was observed between maximal falls of blood pressure by $r$-aminobutyric acid and by $\delta$-aminovaleric acid.

But, between durations of falls by both, there was observed a marked difference.

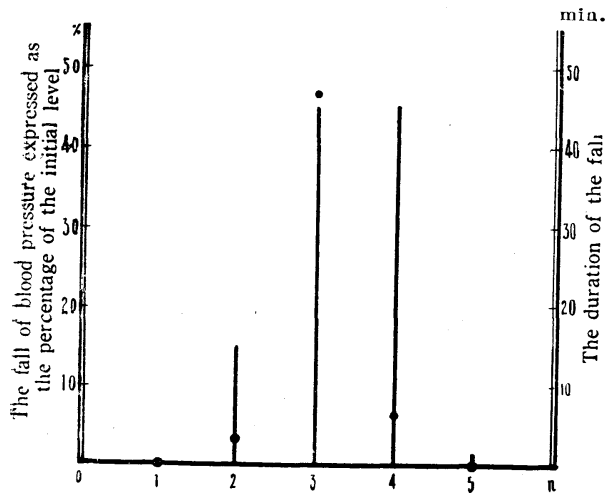

FIG. 6. The relation between the distance of two groups of amino acids and the fall of blood pressure induced by these amino acids.

Abscissa: the percentage magnitude of the fall of blood pressure at the maximum (left). Time in minutes (right).

Coordinate $(n)$ : numbers of carbon atoms between 2 groups.

Black rods: the percentage magnitude of the fall.

Dots: the duration of the fall.

These were the average of two experiments.

Comparison of the effect of $\omega$-amino acids with the effect of $\alpha$-amino acids on blood pressure

Fig. 7 shows the comparison of $\beta$-alanine with $\alpha$-alanine (m.p. $295^{\circ} \mathrm{C}$ ) and $\gamma$-aminobutyric acid with $D L$ - $\alpha$-amino- $n$-butyric acid (m.p. $304^{\circ} \mathrm{C}$., Eastman Kodak Co.) concerning their lowering effect on blood pressure. These data show apparently that the $\alpha$-amino acids with the same length of carbon-atom chains do not affect blood pressure within the limits of concentrations used $(0.05-2.5 \%)$.

Comparison of the effect of $r$-aminobutyric acid with the effect of sodium glutamate on blood pressure

It has been known that in the animal body $r$-aminobutyric acid is produced from glutamic acid by glutamic decarboxylase $(10,11,12)$. The effect of glutamate on the blood pressure was therefore tested.

Fig. 8 shows that an abrupt rise in blood pressure was observed immediately after the injection of glutamate and after the pressure returned to the previous level, a gradual fall took place for a while. But, when the concentration of glutamate was high, blood pressure rose abruptly and, after it once retuned to 

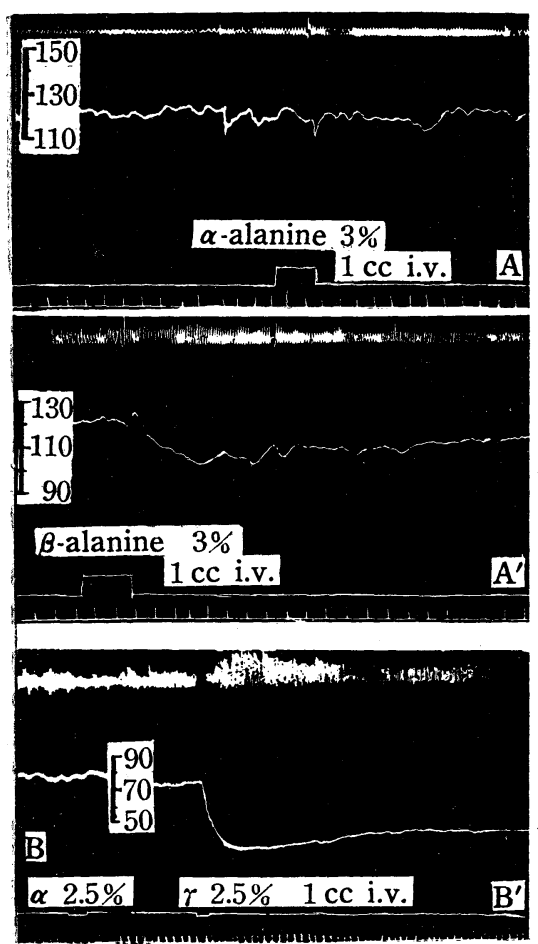

FIG. 8. The effect of glutamate on blood pressure and respiration.

A. Rabbit (2.9 kg.) under urethane anesthesia. R.T. $15^{\circ} \mathrm{C}$.

$1 \mathrm{cc}$. of $8 \%$ sodium glutamate was injected intravenously.

B. Rabbit ( $2.3 \mathrm{~kg}$.) without anesthesia. R.T. $20^{\circ} \mathrm{C}$.

$2.2 \mathrm{cc}$. of $42 \%$ sod. glutamate were injected intravenously. (Time mark $6 \mathrm{sec}$.)
FIG. 7. Comparison of the effect of $\omega^{-}$ amino acids with the effect of $\alpha$-amino acids on blood pressure.

A. $A^{\prime}$. Comparison of the effect of $\alpha$-alanine with the of $\beta \cdot$ alanine.

Rabbit (1.9 kg.) without anesthesia. R.T. $20^{\circ} \mathrm{C}$.

$1 \mathrm{cc}$. each of $3 \% \alpha$-alanine and $3 \% \beta$-alanine was injected intravenously.

B. B'. Comparison of the effect of $\alpha$-amino$n$-butyric acid with that of $\gamma$-amino- $n$-butyric acid.

Rabbit ( $1.8 \mathrm{~kg}$.) under urethane anesthesia. R.T. $20^{\circ} \mathrm{C}$.

1 cc. each of $2.5 \% \alpha$-amino- $n$-butyric acid and $2.5 \% \gamma$-aminobutyric acid was injected intravenously.
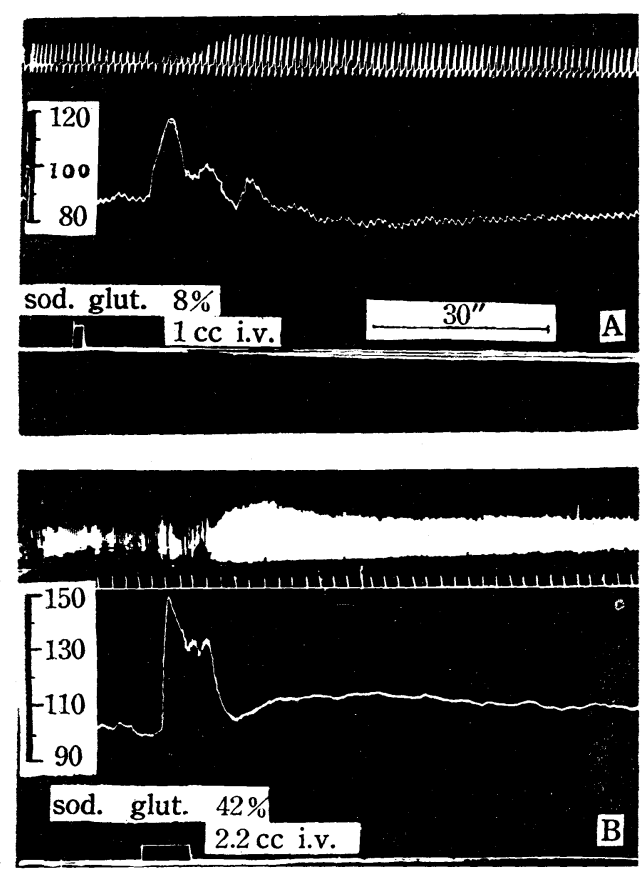
the previous level, it rose slightly again. The latter lasted for a few minutes. The effect of glutamate on respiration was the same as observed with $\gamma$-aminobutyric acid, i.e. at first inhibitory and then acceleratory (Fig. 8).

These results indicate that the depressive action on blood pressure of $\gamma$ aminobutyric acid, is an action characteristic of this acid and not succeeding glutamic acid.

\section{DISCUSSION}

The findings in these experiments indicate that some $\omega$-amino acids cause a drop of blood pressure, and that the distance between amino-group and carboxylic group has an important significance to their effects, that is, the effect of $r$-aminobutyric acid on blood pressure is the most powerful and that of $\beta$-alanine and $\delta$-aminovaleric acid is still considerable, but $\varepsilon$-amino-caproic acid hardly affects blood pressure. It also became clear that corresponding $\alpha$-amino acids do not show any effect on blood pressure, although they have the same length of carbon-atom chains as active $\omega$-amino acids. Consequently, the most striking effect of $\gamma$-aminobutyric acid on blood pressure is due to the fact that the distance between amino group and carboxylic group is optimal.

$\beta$-alanine, which exists in the animal body, also caused a drop of blood pressure. According to Du Vigneaud (4), carnosine, a dipeptide of $\beta$-alanine and histidine, causes a drop of blood pressure. The strength and pattern of their effects on blood pressure seem to be the same by a comparison with our data on $\beta$-alanine with $\mathrm{Du}$ Vigneaud's data on carnosine.

Glutamate causes a rise of blood pressure of the rabbit and this result agrees on the whole with Itoga's result (8) on dogs. This distinct difference between $\gamma$-aminobutyric acid and glutamate enables us to think that the action of $\gamma$ aminobutyric acid on blood pressure is not indirect, but direct.

The relation between the effect and the concentration of $\gamma$-aminobutyric acid was studied in detail. $r$-aminobutyric acid of higher concentrations shows dual effects on blood pressure, i.e. lowering and rising. The rising action of $\gamma$-aminobutyric acid seems to be inhibited due to urethane anesthesia.

The mechanism of the action of $\gamma$-aminobutyric acid on blood pressure, will be discussed in the next paper.

\section{SUMMARY}

All the experiments were performed on rabbits.

1. When $r$-aminobutyric acid was injected intravenously, it caused a fall of blood pressure and some changes in respiration.

The thresholdal dose of $r$-aminobutyric acid affecting blood pressure was less than $70 \mathrm{r} / \mathrm{kg}$. of body weight.

The effect of $\gamma$-aminobutyric acid on blood pressure increased linearly with the logarithm of its concentration. But, in very high concentrations, its effect decreased.

The optimal dose was $8-12 \mathrm{mg} / \mathrm{kg}$. of body weight.

2. $\beta$-alanine and $\delta$-aminovaleric acid also caused fall of blood pressure. But, 
their thresholdal doses were much larger than those of $\gamma$-aminobutyric acid.

$\varepsilon$-aminocaproic acid had only a slight effect and $\alpha$-amino acids no effect on blood pressure.

Glutamate always caused a rise of blood pressure.

3. It was concluded that the depressive action of $\gamma$-aminobutyric acid on blood pressure is due to an optimal distance between the amino group and carboxylic group.

We are indebted to Prof. M. Kubo for his helpful advice and criticism. We are grateful to the Mitsubishi Kasei Institute for the kind donation of $\gamma$-aminobutyric acid, $\beta$ alanine and $\varepsilon$-aminocaproic acid.

This work was supported in part by a research grant from the Department of Education of Japan.

\section{REFERENCES}

1. Awapara, J. Texas Report on Biol. and Medic. 8 : 443-447, 1950.

2. Awapara, J., A. J. Landua, R. Fuerst and B. Seals. J. Biol. Chem. 187: 35-39, 1950.

3. DENT, C. R. AND W. STEPKA. Nature 160: 682, 1947.

4. Du Vigneaud, V. And M. Hunt. J. Biol. Chem. 115: 93-100, 1936.

5. Gilligan, R., J. R. Moor AND S. WARreU. J. Natl. Cancer. Inst. 12: 657, 1951.

6. ITOGA, S. Nihon Seirigaku Zassi 16: 664-672, 1954.

7. Mag, R. M. AND M. J. Thillard. Bull. Soc. Chim. Biol. 33: 253, 1951.

8. MoOR, J. R. AND D. R. Gilligan. J. Natl. Cancer. Inst. 12: 691, 1951 .

9. Robert, E. AND S. Frankel. J. Biol. Chem. 187 : 55-63, 1950.

10. ROBERT, E. AND S. FRANKEL. ibid. 188: 789-795, 1951.

11. ROBERT, E. AND S. FRANKEL. ibid. $190: 505,1951$.

12. Wingo, W. J. AND J. AWApara. ibid. 187: 267-271, 1950. 